\title{
Sur l'optimisation d'actionneurs pour le contrôle d'écoulements
}

\author{
Julien Favier $^{1, a}$, Laurent Cordier $^{2}$ et Azeddine Kourta ${ }^{3}$ \\ 1 « Flubio research team» (Marie-Curie), Università di Genova, DICAT, Via Montallegro 1, 16145 Genova, Italie \\ 2 Chargé de recherche CNRS, LEA, UMR 6609 - CEAT, 43 route de l'aérodrome, 86036 Poitiers Cedex, France \\ 3 Chargé de recherche CNRS, IMFT, UMR 5502, Allée du Professeur Camille Soula, 31400 Toulouse, France
}

Reçu le 15 mars 2007, accepté le 7 juin 2007

\begin{abstract}
Résumé - L'objectif de ce travail est de construire par POD ( Proper Orthogonal Decomposition ») des modèles d'ordre réduit permettant de reproduire de manière fiable la dynamique spatio-temporelle d'un écoulement décollé. L'intérêt croissant pour ces modèles réduits de dynamique s'explique par leur utilisation potentielle dans la résolution de problèmes d'optimisation sous contraintes de grande taille, rencontrés en contrôle d'écoulements. La démarche générale consiste à remplacer le modèle détaillé de l'écoulement (les équations de Navier-Stokes), par un modèle approché, plus rapide à résoudre et contenant les caractéristiques essentielles de la dynamique. La POD, optimale au sens de la reconstruction énergétique, permet d'approximer la dynamique de l'écoulement dans un sous-espace généré par un petit nombre de modes. Différentes méthodes de calibration sont ici développées afin d'améliorer la prédiction du modèle de dimension réduite, et ainsi rendre cette approche utilisable dans le cadre d'une problématique de contrôle d'écoulements. Essentiellement, ces méthodes consistent à ajouter aux équations du modèle des termes supplémentaires calculés comme solutions d'un problème de minimisation sous contraintes.
\end{abstract}

Mots clés : Contrôle d'écoulements / décollement / modèle d'ordre réduit / POD / calibration / optimisation sous contraintes

\begin{abstract}
On the optimization of flow control actuators. The objective of this work is to construct reduced-order models based on POD ("Proper Orthogonal Decomposition") that are able to accurately reproduce the spatio-temporal dynamics of separated flows. The growing interest concerning these models comes from their potential use in the resolution of large scale constrained optimization problems, in the context of flow control. The general approach consists in substituting the high-fidelity model of the NavierStokes equations by an approximate model, cheaper to compute and capturing the essential features of the original dynamics. POD, which is the optimal decomposition in terms of energy, allows to approximate the flow dynamics in a subspace spanned by a few number of modes. Different calibration methods are developed here in order to improve the accuracy of the reduced-order models, and thus make this approach suitable for flow control issues. Essentially, the idea of the calibration methods is to determine in the equations of the POD reduced-order models auxiliary parameters that are computed by resolving appropriate constrained minimization problems.
\end{abstract}

Key words: Flow control / separation / reduced-order model / POD / calibration / constrained optimization

\section{Introduction}

\subsection{Un besoin d'optimisation}

Motivé par une forte demande de la part des industriels du domaine aéronautique et automobile, le contrôle d'écoulements connaît un intérêt scientifique et technologique croissant. D'un point de vue pratique, l'objectif visé est d'améliorer les conditions de fonctionnement

a Auteur correspondant : Julien.Favier@unige.it des engins de transport, en réduisant la consommation de carburant et en augmentant le confort ainsi que la sécurité des passagers [1]. Le contrôle des caractéristiques locales de la couche limite (transition à la turbulence, décollement de couche limite) permet par exemple d'obtenir des gains importants de performances aérodynamiques globales (réduction de traînée, augmentation de portance ou réduction de bruits). En outre, cela constitue un défi scientifique particulièrement stimulant. 


\section{Nomenclature}

\begin{tabular}{|ll|}
\hline$R_{\mathrm{e}}$ & Nombre de Reynolds \\
$N_{\mathrm{t}}$ & Nombre d'échantillons temporels \\
$N_{\text {gal }}$ & Nombre de modes retenu lors de la projection de Galerkin \\
$T$ & Temps de fin de la simulation \\
$\Omega$ & Domaine spatial d'étude \\
$\boldsymbol{x}=(x, y)^{T}$ & Vecteur position d'un point de $\Omega$ \\
$\boldsymbol{u}=(u, v)^{T}$ & Variable de vitesse \\
$\boldsymbol{u}_{\mathrm{m}}=\left(u_{\mathrm{m}}, v_{\mathrm{m}}\right)^{T}$ & Champ moyen de vitesse \\
$p$ & Variable de pression \\
$\boldsymbol{\Phi}_{i}=\left(\phi_{i}^{u}, \phi_{i}^{v}\right)^{T}$ & Fonctions propres spatiales POD d'ordre $i$ \\
$a_{i}(t)$ & Coefficients temporels d'ordre $i$ solutions du modèle réduit POD \\
$\hat{a}_{i}(t)$ & Coefficients temporels de référence d'ordre $i$ évalués par POD \\
$C_{i}$ & Coefficients constants du modèle réduit POD \\
$L_{i j}$ & Coefficients linéaires du modèle réduit POD \\
$Q_{i j k}$ & Coefficients quadratiques du modèle réduit POD \\
$D_{i j}$ & Termes de calibration linéaires \\
$\mathcal{I}$ & Fonctionnelle objectif de la procédure par moindres carrés \\
$\mathcal{J}$ & Fonctionnelle objectif de la procédure d'optimisation sous contraintes \\
$\mathcal{L}$ & $\mathcal{E}_{i}$ \\
$\xi_{i}(t)$ & \\
\hline
\end{tabular}

Depuis près de 20 ans, le contrôle d'écoulements a fait l'objet d'un très grand nombre d'études expérimentales et numériques. La littérature présentant des actionneurs de contrôle d'écoulements est d'ailleurs florissante (se référer à la revue quasi-exhaustive de Gad-el-Hak [2]). Toutefois, un besoin réel d'optimisation de ces moyens d'action demeure, que ce soit pour guider la construction de nouveaux actionneurs basés sur des technologies récentes de matériaux (piézocéramiques, MEMS, ...), ou pour améliorer les rendements énergétiques des mécanismes de contrôle déjà existant. Par conséquent, il est fondamental de déterminer des lois de contrôle optimales, i.e. la meilleure action possible (fréquence de soufflage la plus efficace par exemple) pour un objectif donné (réduire la traînée de forme par exemple). Les études paramétriques simples sont rapidement mises en défaut lorsqu'il s'agit d'un problème mettant en jeu plusieurs paramètres de contrôle (position de l'actionneur, orientation, débit du soufflage, ...). En effet, le calcul de surfaces de réponses de trop grandes dimensions nécessite un coût numérique souvent prohibitif. Il reste cependant possible d'optimiser, à moindre coût, chaque paramètre indépendamment des autres afin d'avoir une idée d'un point de fonctionnement efficace. Mais cette approche ne permet en aucun cas de conclure sur le point de fonctionnement optimal, ce qui est fondamental dans le cadre de problématiques industrielles dans lesquelles la notion de rendement énergétique est prioritaire.

\subsection{Complexité de calcul}

Pour résoudre ce type de problèmes d'optimisation sous contraintes, une méthode vient naturellement à l'esprit : le contrôle optimal [3], mais son utilisation s'accompagne d'un certain formalisme mathématique et le coût numérique est généralement important.

Le contrôle optimal est basé sur la minimisation d'une fonctionnelle objectif (le coefficient de traînée, le bruit, ...) par rapport à certains paramètres de contrôle (débit ou fréquence d'un actionneur de soufflage par exemple), sous les contraintes des équations du mouvement, i.e. les équations de Navier-Stokes. Pour résoudre ce problème, deux grandes voies sont possibles, d'une part, les méthodes de type descente qui nécessitent au minimum une approximation du gradient de la fonctionnelle objectif, et, d'autre part, les méthodes stochastiques qui étudient par exemple l'évolution d'une population d'individus au cours 
de générations successives (algorithmes génétiques). Dans les deux approches, les équations du mouvement (NavierStokes) doivent être résolues un grand nombre de fois, ce qui s'accompagne in fine de coûts numériques prohibitifs dans le cadre de problématiques réalistes de contrôle d'écoulements.

\subsection{Une solution}

Dans le but de contourner ce problème, la modélisation de dimension réduite constitue un outil puissant, permettant une approximation de la dynamique d'un écoulement sur un espace de faible dimension. La mise en œuvre du contrôle optimal en est alors facilitée car les temps de calculs et les stockages mémoires associés sont alors considérablement réduits [4].

Dans cette optique de compression d'informations, la POD ou Proper Orthogonal Decomposition [5] permet d'extraire une base de l'écoulement optimale au sens de la reconstruction énergétique. La projection de Galerkin des équations de Navier-Stokes sur un nombre fini de modes POD permet alors d'obtenir un système d'équations différentielles ordinaires en temps : le modèle d'ordre réduit POD qui peut être construit en formulation incompressible [4-7] ou compressible [8]. La résolution de ce système d'équations donne l'évolution temporelle de la dynamique de l'écoulement restreinte au sous-espace POD. Ce modèle peut alors être utilisé en remplacement des équations de Navier-Stokes dans le cadre de la résolution d'un problème d'optimisation.

Cependant, en raison de la troncature réalisée dans l'espace des modes, ce modèle réduit de dynamique peut ne pas être suffisamment précis. En effet, puisque la dissipation s'effectue au niveau des petites échelles de l'écoulement, les modes POD peu énergétiques d'ordre élevé, qui ont été négligés dans la construction du modèle, jouent cependant un rôle important. Afin de corriger ce manque de dissipation inhérent à l'approche POD-Galerkin, des méthodes de calibration sont développées. Essentiellement, des termes de différentes natures (constants, linéaires ou visqueux) sont ajoutés au système afin d'améliorer la qualité de l'approximation de la dynamique de référence. Ces termes sont calculés, soit par moindres carrés, soit en résolvant un problème d'optimisation contraint visant à minimiser l'écart entre la dynamique calculée par le modèle et la dynamique de référence, sous réserve que les termes de calibration vérifient les équations du modèle d'ordre réduit POD.

La construction du modèle, ainsi que la présentation des différentes méthodes de calibration, sont tout d'abord effectuées pour une dynamique d'écoulement relativement classique : l'écoulement derrière un cylindre circulaire à $R_{\mathrm{e}}=200$, simulé par DNS (code Icare, IMFT [9]). La méthodologie de construction d'un modèle d'ordre réduit POD calibré est ensuite appliquée au cas d'un écoulement décroché autour d'un profil NACA012 à $R_{\mathrm{e}}=5000$, simulé par DNS (code commercial Fluent). Ces deux configurations sont respectivement qualifiées dans la suite de cylindre-DNS et profil-DNS.

\section{Obtention du modèle POD}

Partant d'un jeu de $N_{\mathrm{t}}$ grandeurs spatiotemporelles (vitesse, pression, ...), mesurées ou simulées numériquement, la méthode des snapshots [4] permet de déterminer une base POD représentative de ces variables. La vitesse, par exemple, s'exprime au moyen de cette décomposition comme un produit de fonctions propres spatiales $\boldsymbol{\Phi}_{i}(\boldsymbol{x})$ et temporelles $a_{i}(t)$ :

$$
\boldsymbol{u}(\boldsymbol{x}, t)=\boldsymbol{u}_{\mathrm{m}}(\boldsymbol{x})+\sum_{i=1}^{N_{\mathrm{t}}} a_{i}(t) \boldsymbol{\Phi}_{i}(\boldsymbol{x})
$$

où $\boldsymbol{u}_{\mathrm{m}}$ représente la moyenne d'ensemble des $N_{\mathrm{t}}$ échantillons de vitesse et où $\boldsymbol{x} \in \Omega$ est la variable d'espace.

Le modèle réduit POD est ensuite obtenu par projection de Galerkin des équations de Navier-Stokes sur le sous-espace généré par un petit nombre $N_{\text {gal }}$ de modes POD. Soit $(., .)_{\Omega}$, un produit scalaire de l'espace $\Omega$ dans $\mathcal{L}^{2}$, l'espace des fonctions de carré intégrable, la projection de Galerkin s'écrit formellement :

$$
\left(\boldsymbol{\Phi}_{i}, \frac{\partial \boldsymbol{u}}{\partial t}+(\boldsymbol{u} \cdot \vec{\nabla}) \boldsymbol{u}\right)_{\Omega}=\left(\boldsymbol{\Phi}_{i},-\vec{\nabla} p+\frac{1}{R_{\mathrm{e}}} \Delta \boldsymbol{u}\right)_{\Omega}
$$

En introduisant la décomposition (1) dans la projection (2), on obtient, après quelques manipulations algébriques, le système d'équations différentielles ordinaires suivant :

$$
\left\{\begin{aligned}
\frac{\mathrm{d} a_{i}}{\mathrm{~d} t} & =C_{i}+\sum_{j=1}^{N_{\mathrm{gal}}} L_{i j} a_{j}+\sum_{j=1}^{N_{\mathrm{gal}}} \sum_{k=1}^{N_{\mathrm{gal}}} Q_{i j k} a_{j} a_{k}+\left(\boldsymbol{\Phi}_{i},-\vec{\nabla} p\right)_{\Omega} \\
a_{i}(0) & =\left(\boldsymbol{u}(\boldsymbol{x}, 0)-\boldsymbol{u}_{\mathrm{m}}(\boldsymbol{x}), \boldsymbol{\Phi}_{i}(\boldsymbol{x})\right)_{\Omega}
\end{aligned}\right.
$$

Les coefficients constants $C_{i}$, linéaires $L_{i j}$, et quadratiques $Q_{i j k}$ dépendent explicitement de $\boldsymbol{\Phi}_{i}, \boldsymbol{u}_{\mathrm{m}}$ et de $R_{\mathrm{e}}$. Selon l'approche classique POD-Galerkin, ces coefficients sont calculés une fois pour toute au début de la procédure. Le système d'équations du modèle POD d'ordre réduit est alors intégré en temps par un schéma Runge-Kutta d'ordre 4, ce qui donne l'évolution temporelle des coefficients $a_{i}(t)$ prédite par le modèle (la dynamique spatiale étant capturée par les modes spatiaux $\left.\Phi_{i}(\boldsymbol{x})\right)$. Afin de tester la qualité de l'approximation réalisée, les coefficients $a_{i}(t)$ sont comparés avec les coefficients temporels POD $\hat{a}_{i}(t)$ directement déterminés à partir du jeu d'échantillons de référence.

Comme dans la majorité des travaux de la littérature qui s'intéressent à des configurations incompressibles [10 par exemple], la contribution du terme de pression $\left(\boldsymbol{\Phi}_{i},-\vec{\nabla} p\right)_{\Omega}$ est tout simplement négligée. Dans le cas cylindre-DNS, la dynamique temporelle, calculée en résolvant le système (3), n'est pas parfaitement prédite. La figure 1 représente la comparaison de la dynamique temporelle prédite par le modèle réduit POD pour $N_{\text {gal }}=6$ i.e. en prenant en compte les six premiers modes 

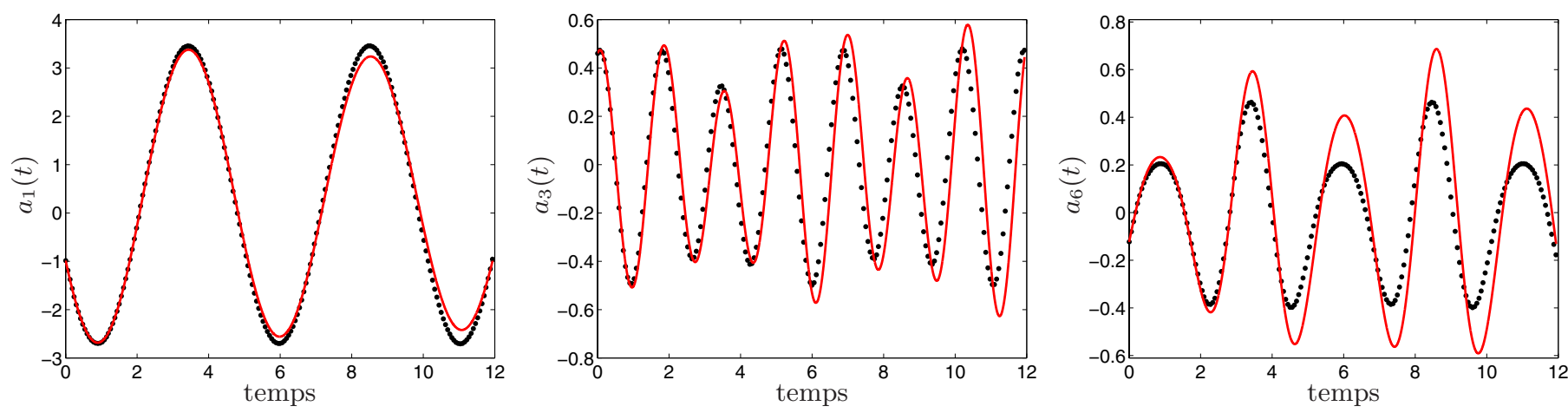

Fig. 1. Comparaison des coefficients temporels calculés par le modèle réduit sans calibration aux coefficients temporels POD. Cas cylindre-DNS. — dynamique temporelle prédite par le modèle réduit POD ; ... dynamique temporelle de référence.
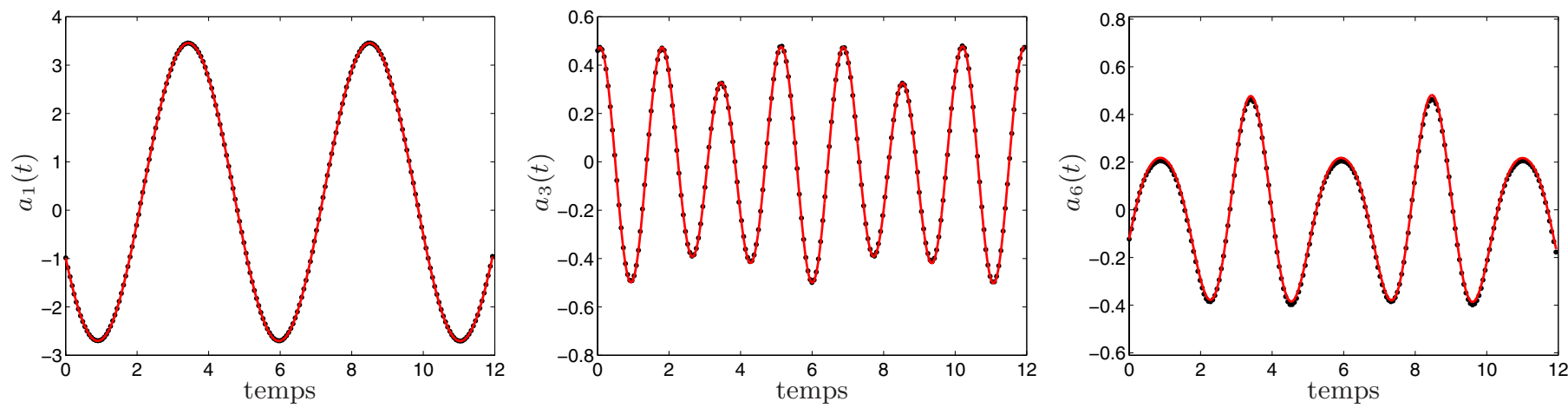

Fig. 2. Comparaison des coefficients temporels calculés par le modèle réduit calibré aux coefficients temporels POD. Cas cylindre-DNS. - dynamique temporelle prédite par le modèle réduit POD, ... dynamique temporelle de référence.

POD, soit $99 \%$ de l'énergie cinétique de l'écoulement, avec la dynamique de référence obtenue par POD. Comme le montre cette figure, les maxima de référence ont tendance à être surestimés (ce qui est lié au manque de dissipation du modèle) et un léger déphasage en temps apparaît. Globalement, les erreurs d'approximation sont plus marquées sur les coefficients d'ordre élevé.

Afin d'améliorer la prédiction du modèle, différentes méthodes de calibration sont introduites à la section 3 pour le cas cylindre-DNS. Une application est ensuite réalisée à la section 4 sur la configuration profil-DNS.

\section{Calibration des modèles POD}

Essentiellement, les méthodes de calibration consistent à ajouter au système (3) des termes supplémentaires. Selon la méthode retenue, ces termes peuvent correspondre à des coefficients linéaires [11], des coefficients constants et linéaires [12], ou des viscosités tourbillonnaires déterminées de manière optimale pour chaque mode [4]. La section suivante présente des méthodes de calcul pour ces différents termes.

\section{Procédure de moindres carrés}

Cette première méthode correspond à une minimisation par moindres carrés de l'écart entre les coefficients temporels $a_{i}$ calculés par le modèle réduit POD, et les coefficients temporels $\hat{a}_{i}$ déterminés aux instants $t_{k}$ à partir du jeu d'échantillons de référence, soit :

$$
\hat{a}_{i}\left(t_{k}\right)=\left(\boldsymbol{u}\left(\boldsymbol{x}, t_{k}\right)-\boldsymbol{u}_{\mathrm{m}}(\boldsymbol{x}), \boldsymbol{\Phi}_{i}\right)_{\Omega}
$$

Ce problème peut se ramener à la minimisation de la fonctionnelle $\mathcal{I}$ suivante :

$$
\mathcal{I}=\sum_{i=1}^{N_{\text {gal }}} \sum_{s=1}^{N_{\mathrm{t}}}\left[\dot{a}_{i}\left(t_{\mathrm{s}}\right)-\dot{\hat{a}}_{i}\left(t_{\mathrm{s}}\right)\right]^{2}
$$

Soit $D_{i j}$ un terme linéaire de calibration, une expression analytique de celui-ci peut être déterminée en imposant que la dérivée $\frac{\partial \mathcal{I}}{\partial D_{i j}}$ s'annule. Le calcul de termes de calibration constants ou visqueux, se fait de manière strictement identique [5]. Une simple résolution d'un système linéaire matriciel permet alors le calcul de ces termes. La calibration du modèle par des termes linéaires par exemple (Fig. 2), permet de corriger les surestimations des maxima et les déphasages temporels observés sur la figure 1 . 


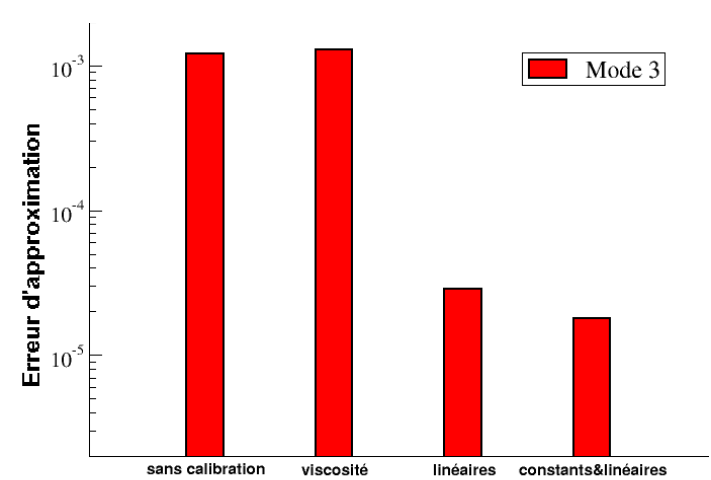

Type de termes de calibration

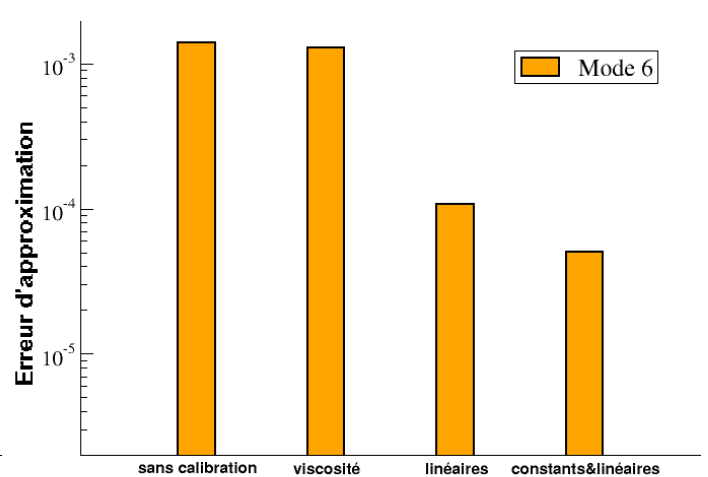

Type de termes de calibration

Fig. 3. Variation dans le cas cylindre-DNS de l'erreur d'approximation selon la forme des termes de calibration utilisés.

Afin de comparer l'efficacité des différentes formes de termes de calibration, l'erreur d'approximation $\mathcal{E}_{i}$ est introduite selon la définition suivante :

$$
\mathcal{E}_{i}=\frac{1}{N_{\mathrm{t}}} \sqrt{\sum_{s=1}^{N_{\mathrm{t}}}\left(a_{i}\left(t_{\mathrm{s}}\right)-\hat{a}_{i}\left(t_{\mathrm{s}}\right)\right)^{2}} .
$$

La figure 3 présente les erreurs d'approximation associées aux différentes formes de termes de calibration. Les meilleurs résultats sont obtenus dans le cas où la calibration est effectuée par ajout de termes constants et linéaires. En effet, par rapport à l'ajout d'une viscosité sur chaque mode [4], les termes linéaires $D_{i j}$ prennent en compte les interactions intermodales par l'intermédiaire des termes non diagonaux [5]. En ce sens, ils constituent un moyen plus complet de calibration. Dans la suite, les méthodes de calibration seront basées sur l'introduction de termes constants et linéaires.

\section{Procédure d'optimisation sous contraintes}

Bien que la précédente méthode fournisse des résultats très bons dans le cas cylindre- $D N S$, il peut être nécessaire, dans le cas de configurations d'écoulements plus complexes, de faire appel à une méthode mathématiquement plus rigoureuse (optimisation sous contraintes [3]). Soit $T$ l'instant de fin de la simulation, le principe consiste à déterminer les termes de calibration $D_{i j}$ qui minimisent la fonctionnelle $\mathcal{J}$ suivante :

$$
\mathcal{J}\left(a_{i}, D_{i j}\right)=\int_{0}^{T} \sum_{i=1}^{N_{\text {gal }}}\left[a_{i}(t)-\hat{a}_{i}(t)\right]^{2} \mathrm{~d} t
$$

sous les contraintes des équations calibrées (3). Par rapport à la méthode précédente, en plus de reconstruire l'information provenant des coefficients de référence $\hat{a}_{i}$, on impose aussi de respecter les équations du mouvement, ce qui en fait une méthode plus satisfaisante mathématiquement. La résolution de ce problème est effectuée par la méthode des multiplicateurs de Lagrange où les contraintes sont imposées via l'introduction d'une

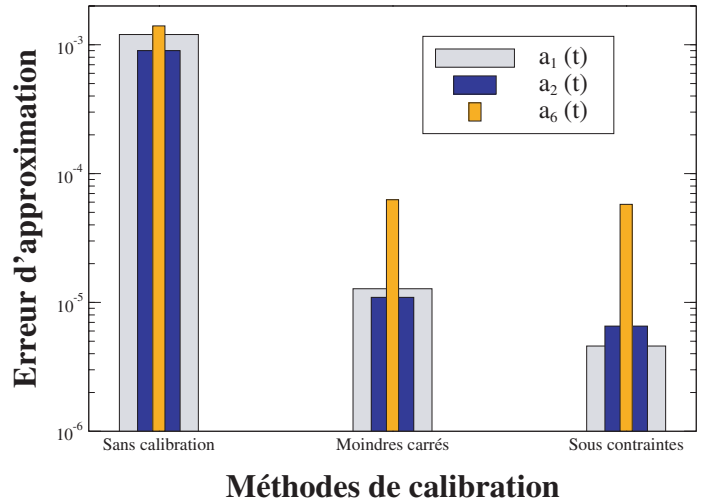

Fig. 4. Comparaison des erreurs d'approximation obtenues par les différentes méthodes de calibration pour le cas cylindreDNS.

nouvelle fonctionnelle $\mathcal{L}$, par l'intermédiaire de variables adjointes $\boldsymbol{\xi}$ :

$\mathcal{L}\left(a_{i}, D_{i j}, \xi_{k}\right)=\mathcal{J}\left(a_{i}, D_{i j}\right)-\sum_{k=1}^{N_{\text {gal }}} \int_{0}^{T} \xi_{k}(t) \mathcal{N}_{k}\left(a_{i}, D_{i j}\right) \mathrm{d} t$.

Dans cette expression, les contraintes $\mathcal{N}_{k}\left(a_{i}, D_{i j}\right)=0$ correspondent à l'équation $k$ du modèle calibré (3). En considérant que chaque variable de $\mathcal{L}$ est indépendante des autres (ce qui est faux en toute rigueur), le système optimal associé au problème est déterminé en annulant successivement la dérivée directionnelle de $\mathcal{L}$ par rapport aux variables $\boldsymbol{\xi}_{k}, a_{i}$ et $D_{i j}$ :

- imposer $\frac{\partial \mathcal{L}}{\partial \boldsymbol{\xi}_{k}}=0$ redonne, par construction de la fonctionnelle $\mathcal{L}$, les équations d'état $\mathcal{N}_{k}\left(a_{i}, D_{i j}\right)=0$,

- imposer $\frac{\partial \mathcal{L}}{\partial a_{i}}=0$ donne les équations adjointes, et

- imposer $\frac{\partial \mathcal{L}}{\partial D_{i j}}=0$ donne finalement la condition d'optimalité, ce qui permet d'évaluer le gradient de la fonctionnelle coût par rapport aux paramètres de contrôle.

Comme il est fait mention dans l'introduction à propos des méthodes du type descente, la résolution du système constitué de ces trois équations est souvent extrêmement lourde numériquement. Cependant, dans ce cas, les équations d'état et adjointes étant restreintes au 


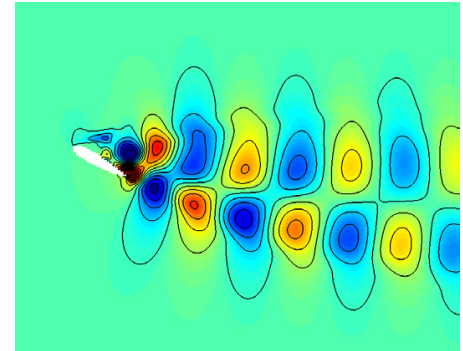

(a) mode $\Phi_{1}$

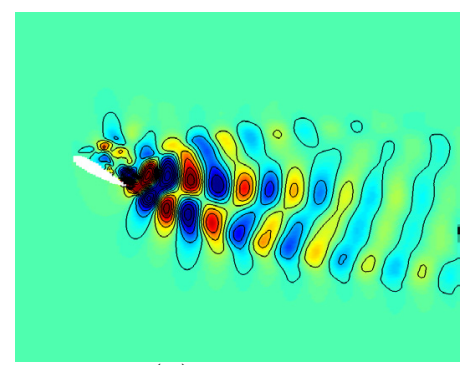

(d) mode $\Phi_{4}$

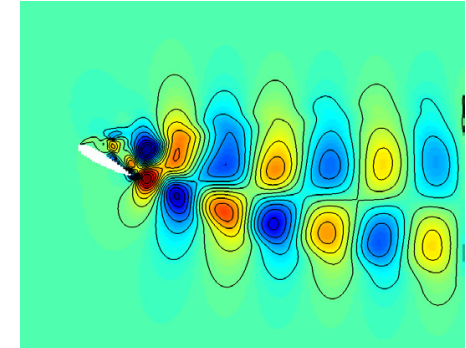

(b) mode $\Phi_{2}$

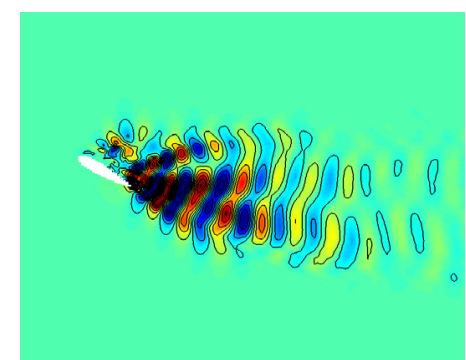

(e) mode $\Phi_{5}$

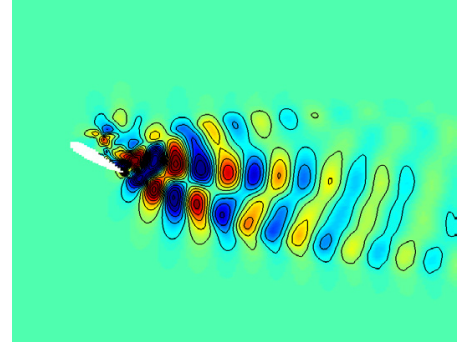

(c) mode $\Phi_{3}$

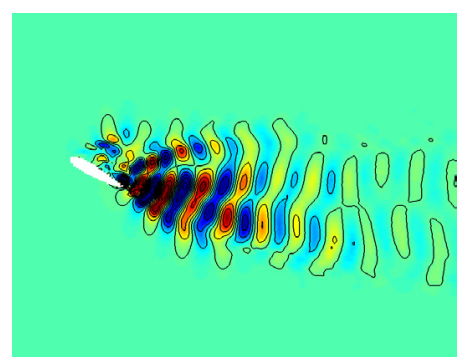

(f) mode $\Phi_{6}$

Fig. 5. Premiers modes POD spatiaux de la vitesse longitudinale dans le cas profil-DNS.

sous-espace réduit POD, leur résolution est peu coûteuse, allégeant ainsi la procédure globale en termes de temps de calcul et de stockage mémoire.

La figure 4 compare différentes méthodes de calibration de termes constants et linéaires dans le cas cylindre$D N S$. Il apparait que la méthode d'optimisation sous contraintes affiche des résultats meilleurs que la procédure par moindres carrés. Cette approche, plus satisfaisante, permet en effet d'obtenir une calibration plus efficace, la différence se faisant essentiellement sur les premiers modes, les plus énergétiques par construction.

Pour résumer, la calibration la plus efficace est donc effectuée par ajout de termes constants et linéaires, calculés en résolvant le problème d'optimisation sous contraintes présenté ci-dessus. Cette méthode est appliquée dans la section suivante au cas d'un écoulement décollé autour d'un profil d'aile.

\section{Application}

Le cas profil-DNS correspond à une configuration d'écoulement décroché autour d'un profil NACA012. Le nombre de Reynolds $R_{\mathrm{e}}$ est fixé à 5000 et l'angle d'attaque à $16^{\circ}$. Les simulations sont réalisées par DNS (code Fluent). Les modes POD sont extraits d'une base de $N_{\mathrm{t}}$ échantillons capturant à la fois la zone décollée autour de l'extrados, ainsi que le sillage derrière le profil. Comme on peut le voir sur la figure 5, les modes spatiaux sont organisés par paires associées aux mêmes gammes d'échelle. Leurs structures rappellent celles des modes calculés pour l'écoulement derrière un cylindre circulaire dont le sillage est dominé par les allées de Von Kármán. Clairement, la POD permet ici d'extraire les informations relatives à la périodicité du détachement tourbillonnaire s'effectuant dans le sillage du profil. Il est donc raisonnable de penser qu'un modèle de dimension réduite pourra reconstruire efficacement l'écoulement avec peu de modes POD.

Le modèle réduit est construit sur 8 modes (ce qui représente $99 \%$ de l'énergie cinétique de l'écoulement dans ce cas) selon l'approche POD-Galerkin décrite à la section 2. La calibration est effectuée par ajout de termes constants et linéaires, calculés en résolvant un problème de minimisation sous contraintes. Comme l'illustre la figure 6 , le modèle non calibré se révèle plus instable que dans le cas cylindre-DNS. En effet, les maxima sont fortement surestimés, spécialement pour les coefficients d'ordre élevé et le modèle tend à diverger aux temps longs. La calibration permet par contre de corriger les erreurs d'approximation et l'on obtient finalement un modèle capable de reconstruire correctement la dynamique temporelle de référence.

\section{Conclusion et perspective directe}

Le principe de la démarche suivie dans cet article est résumé schématiquement sur la figure 7. Partant des équations de Navier-Stokes continues, des solutions sont d'abord obtenues dans un espace discrétisé. La méthode POD-Galerkin permet ensuite de compresser l'information et de construire un modèle de dimension réduite reproduisant les caractéristiques essentielles de la dynamique. Finalement, la calibration permet de corriger les erreurs d'approximation intrinsèques du modèle pour le rendre plus fidèle à la dynamique de référence.

Avec cette méthodologie et les techniques de calibration présentées ici, des modèles d'ordre réduit POD peuvent donc reproduire de manière fiable la dynamique 


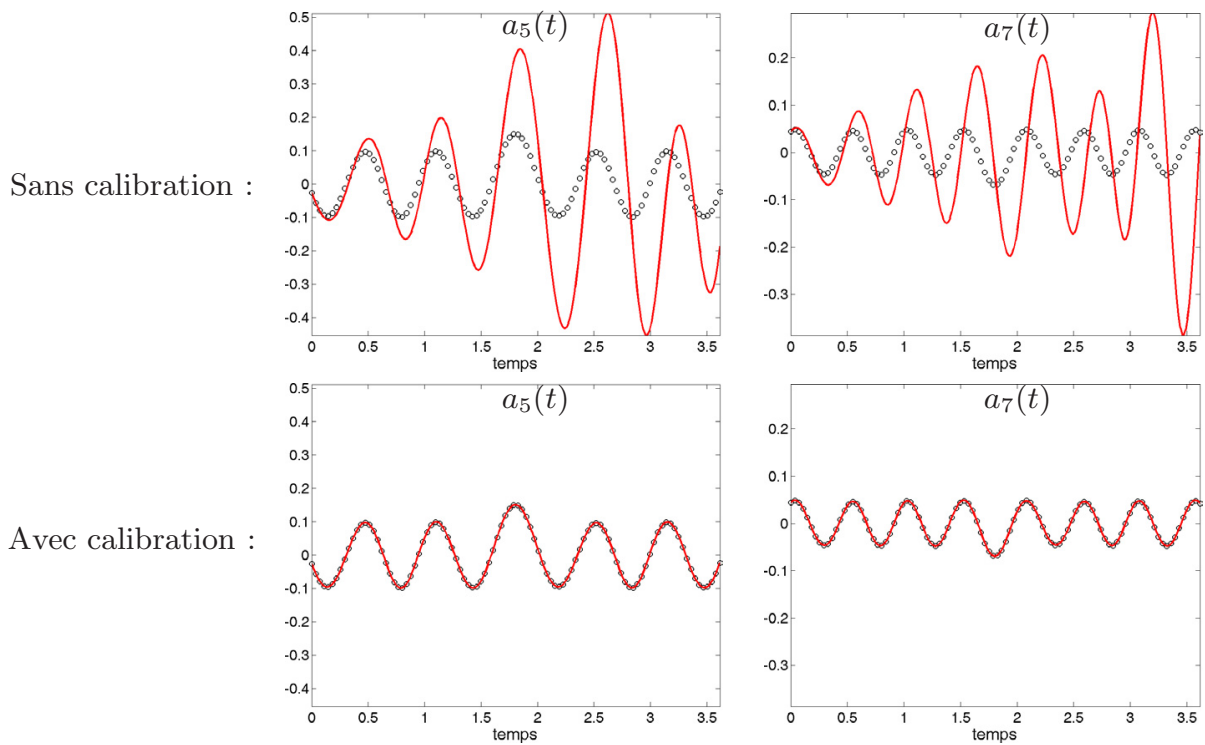

Fig. 6. Comparaison des coefficients temporels calculés par le modèle réduit avec et sans calibration aux coefficients temporels POD. Cas profil-DNS. — dynamique temporelle prédite par le modèle réduit POD, ... dynamique temporelle de référence.

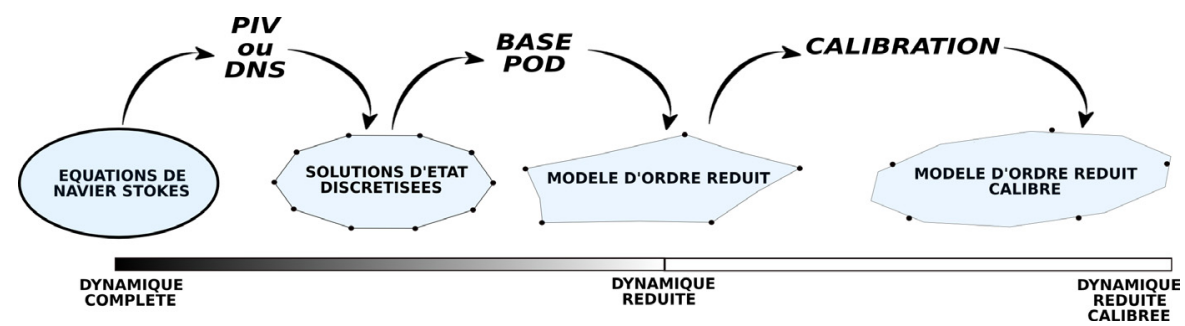

Fig. 7. Synthèse de la démarche.

d'un écoulement décollé présentant des détachements tourbillonnaires périodiques. Ces résultats encourageants permettent d'envisager le calcul de lois de contrôle optimales à partir de ces modèles [4]. Cet aspect constitue la perspective directe de cette étude.

Ce travail résulte d'une collaboration au sein du GDR 2502 «Contrôle des décollements ». Le premier auteur remercie le projet Marie-Curie « FLUBIO » MEST-CT-2005-020228 pour son soutien financier.

\section{Références}

[1] P. Gilliéron, E. Levallois-Goy, Contribution de l'aérodynamique au développement durable et sécuritaire dans l'automobile, Mécanique \& Industries 6 (2006) $567-574$

[2] M. Gad-el-Hak, Flow Control, Passive, Active and Reactive Management, Cambridge University Press, 2004

[3] M.D. Gunzburger, Introduction into mathematical aspects of flow control and optimization, Lecture series 1997-05 on inverse design and optimization methods, Von Kármán Institute for Fluid Dynamics, 1997

[4] M. Bergmann, Optimisation aérodynamique par réduction de modèle $\mathrm{POD}$ et contrôle optimal. Application au sillage laminaire d'un cylindre circulaire, Thèse de doctorat, Institut National Polytechnique de Lorraine, 2004
[5] J. Favier, Contrôle d'écoulements : approche expérimentale et modélisation de dimension réduite, Thèse de doctorat, Institut National Polytechnique de Toulouse, 2007

[6] L. Perret, Étude du couplage instationnaire calculsexpériences en écoulements turbulents, Thèse de doctorat, Université de Poitiers, 2004

[7] M. Couplet, Modélisation POD-Galerkine réduite pour le contrôle des écoulements instationnaires, Thèse de doctorat, Université Paris XIII, 2005

[8] G. Vigo, Méthodes de décomposition orthogonale aux valeurs propres appliquées aux écoulements instationnaires compressibles complexes, Thèse de doctorat, Université Paris IX Dauphine, 2000

[9] M. Braza, P. Chassaing, H. Ha Minh, Numerical study and physical analysis of the pressure and velocity fields in the near wake of a circular cylinder, J. Fluid Mech. 165 (1986) 79-130

[10] B.R. Noack, P. Papas, P.A. Monkewitz, The need for a pressure-term representation in empirical Galerkin models of incompressible shear flows, J. Fluid Mech. 523 (2005) 339-365

[11] B. Galletti, C.-H. Bruneau, L. Zanetti, A. Iollo, Loworder modelling of laminar flow regimes past a confined square cylinder, J. Fluid Mech. 503 (2004) 161-170

[12] M. Buffoni, S. Camarri, A. Iollo, M.V. Salvetti, Lowdimensional modelling of a confined three-dimensional wake flow, J. Fluid Mech. 569 (2006) 141-150 